\title{
SOME RECENT MEASUREMENTS OF PROTON FORM FACTORS
}

\author{
W. Albrecht, H.-J. Behrend, H. Dorner, W. Flauger, and H. Hultschig \\ Deutches Elektronen-Synchrotron, Hamburg, Germany
}

(Received 24 April 1967)

An account was given earlier ${ }^{1}$ on measurements of proton form factors up to very high momentum transfers. In the following the results of some further measurements are related which were made with the same experimental setup.

Electrons were scattered elastically from an internal hydrogen target in the Deutches Elektronen-Synchrotron (DESY) 6-GeV electron synchrotron. They were momentum analyzed in a doubly focusing spectrometer which was composed of two quadrupoles and one bending magnet. An array of scintillation counters in the focus of the optical system provided the momentum definition. Electrons were identified by a threshold Čerenkov counter and a shower counter. In the $q^{2}=245 \mathrm{~F}^{-2}$ run the counting rate was only about 2 per hour, and special care had to be taken to eliminate background particles (accidental coincidences or pions); the pulses of the counters were photographed in order to eliminate false events which had not been recognized by the logic. In addition, the pulses were channeled into a time to pulseheight converter (TPC) system which only rendered a maximum pulse in the case of optimum overlap of all pulses. The pulse-time resolution of the logic was improved from $2 \tau=17 \mathrm{nsec}$ to $2 \tau=6$ nsec by pulse-height discrimination of the TPC output.

Calibration of the measurements was done by counting elastic recoil protons of $q^{2}=13 \mathrm{~F}^{-2}$. In this way difficulties were avoided which are inherent to the more usual calibration for internal-target experiments by the quantameter method, in which uncertainties are introduced by the bremsstrahlung from the target cup. The exact method is described in Ref. 1 .
The results are given in Table I. The magnetic form factor for $q^{2}=245 \mathrm{~F}^{-2}$ could be taken directly from the cross-section measurement at $\theta=75^{\circ}$, since the contribution to the cross section due to the electric form factor is only approximately $1 \%$, assuming $G_{M} / \mu=G_{E}$. The data for $q^{2}=50 \mathrm{~F}^{-2}$ and $75 \mathrm{~F}^{-2}$ were combined with those of Bartel et al. ${ }^{2}$ Form factors for these $q^{2}$ were obtained by means of the Rosenbluth straight line. The precision is better than that of former measurements; within the error limits the relation $G_{E}=(1 / \mu) G_{M}$ is confirmed.

In Fig. 1 the data on the electric form factor including those from this experiment are shown. For comparison, the well-known dipole fit, which was derived from measurements of the magnetic form factor, is plotted as well. Up to $q^{2}=45 \mathrm{~F}^{-2}$ all available data were evaluated. ${ }^{1-7}$ The form factors were calculated from the Rosenbluth straight-line fit to all measured cross sections for each momentum transfer. Above $q^{2}=45 \mathrm{~F}^{-2}$ only the data taken at DESY are included.

Figure 2 shows the magnetic form factor in logarithmic representation. In particular, the form-factor dependence in the whole region is satisfactorily represented by the simple dipole fit

$$
G_{M} / \mu=\left(1+q^{2} / 0.71\right)^{-2}
$$

If a hard core exists in the electromagnetic structure of the proton, its $G_{M} / \mu$ would have to be smaller than $4 \times 10^{-3}$.

Attempts are being made at present to fit the measured points by pole formulas or exponential terms. The results of this work will

Table I. Cross sections and form factors deduced from this experiment.

\begin{tabular}{|c|c|c|c|c|c|c|}
\hline $\begin{array}{c}q^{2} \\
\left(\mathrm{~F}^{-2}\right)\end{array}$ & $\begin{array}{c}E_{0} \\
(\mathrm{GeV})\end{array}$ & $\begin{array}{c}\theta_{e} \\
(\operatorname{deg})\end{array}$ & $\begin{array}{c}d \sigma / d \Omega \\
\left(\mathrm{cm}^{2} / \mathrm{sr}\right)\end{array}$ & $G_{E}$ & $G_{M} / \mu$ & Evaluation \\
\hline 50.0 & 1.77 & 75.87 & $(1.41 \pm 0.06) \times 10^{-34}$ & $0.075_{-0.018}^{+0.014}$ & $0.074 \pm 0.003$ & \multirow{3}{*}{$\begin{array}{l}\text { This work combined } \\
\text { with Bartel et al. } \\
G_{E}=G_{M} / \mu\end{array}$} \\
\hline 75.0 & 2.37 & 75.83 & $(2.76 \pm 0.12) \times 10^{-35}$ & $0.036_{-0.027}^{+0.014}$ & $0.0411 \pm 0.0015$ & \\
\hline 245.5 & 6.13 & 75.78 & $(6.04 \pm 0.85) \times 10^{-38}$ & ... & $0.0043 \pm 0.0003$ & \\
\hline
\end{tabular}

\footnotetext{
${ }^{\mathrm{a}} \mathrm{Ref.} 2$.
} 


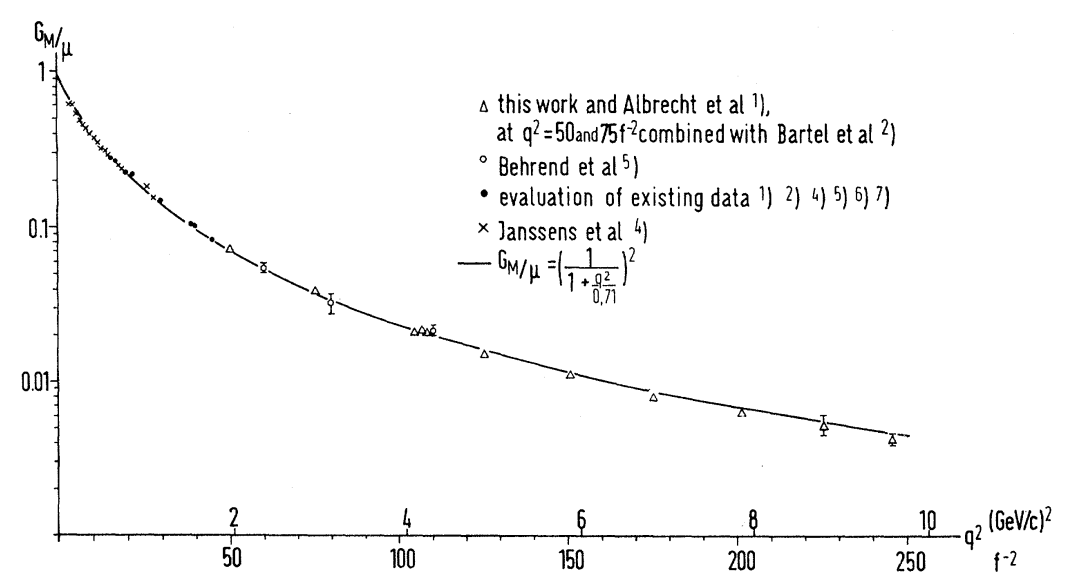

FIG. 1. Electric form factor versus $q^{2}$. For comparison also the dipole fit deduced from the magnetic form factor is plotted.

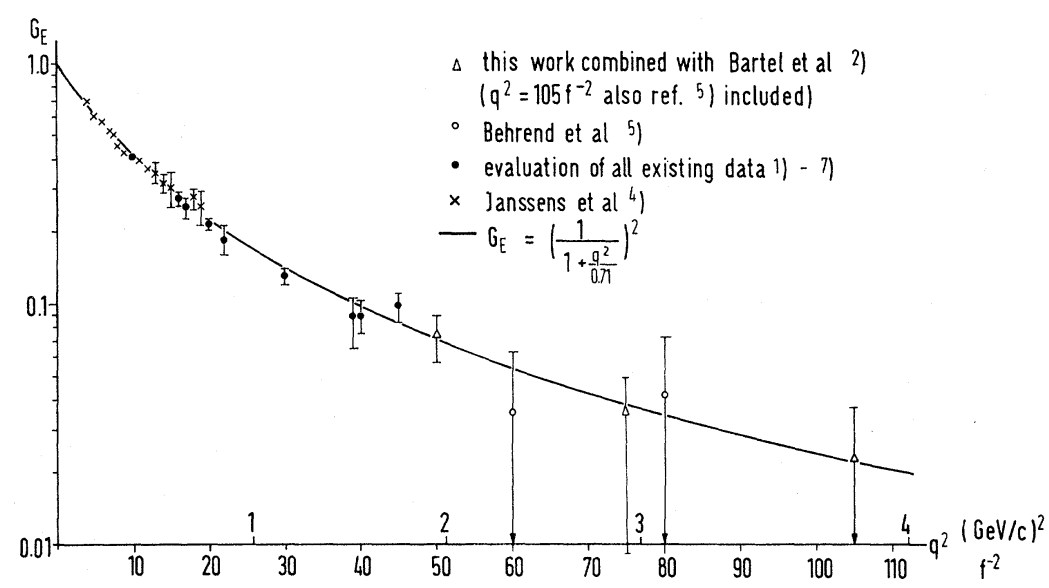

FIG. 2. Magnetic form factor versus $q^{2}$.

be related elsewhere.

We are greatly indebted to Professor W. Jentschke and Professor P. Stähelin for their interest and encouragement, and to the machine group of DESY for the excellent performance of the synchrotron. The Čerenkov counter was built by S. Galster and G. Hartwig of the Institut fur Experimentelle Kernphysik, Kernforschungszentrum Karlsruhe.

${ }^{1}$ W. Albrecht, H.-J. Behrend, F. W. Brasse, W. Flauger, H. Hultschig, and K. G. Steffen, Phys. Rev. Letters 17,1192 (1966).

${ }^{2}$ W. Bartel, B. Dudelzak, H. Krehbiel, J. M. McEl- roy, U. Meyer-Berkhout, R. J. Morrison, H. NguyenNgoc, W. Schmidt, and G. Weber, Phys. Rev. Letters 17, 608 (1966).

${ }^{3} \mathrm{~B}$. Dudelzak, A. Isakov, P. Lehmann, and R. Tchapoutian, in Proceedings of the Twelfth International Conference on High Energy Physics, Dubna, 1964 (Atomizdat., Moscow, 1966), Vol. I, p. 916.

${ }^{4}$ T. Janssens, R. Hofstadter, E. B. Hughes, and M. R. Yearian, Phys. Rev. 142, 922 (1966).

${ }^{5}$ H.-J. Behrend, F. W. Brasse, J. Engler, S. Galster, E. Ganssauge, G. Hartwig, H. Hultschig, and H. Schopper, Deutches Elektronen-Synchrotron Report No. DESY 66/27, 1966 (unpublished).

${ }^{6}$ K. W. Chen, J. R. Dunning, A. A. Cone, N. F. Ramsey, J. K. Walker, and R. Wilson, Phys. Rev. 141, 1267 (1966).

${ }^{7}$ K. Berkelman, M. Feldman, R. M. Littauer, G. Rouse, and R. R. Wilson, Phys. 130, 2061 (1963). 